\title{
Abstracts of the Symposium on Neuropsychological Rehabilitation, 12-13 July, 2004, Uluru, Australia:
}
A Satellite Symposium to the Joint 27th Conference of the Australian Society for the Study of Brain Impairment and the 27th Mid-year Meeting of the International Neuropsychological Society, 7-10 July, 2004, Brisbane.

\section{Organising Committee}

UK Committee

Barbara Wilson, (Convenor), Medical Research Council, Cognition and Brain Sciences Unit, Cambridge and The Oliver Zangwill Centre, Ely

Camilla Herbert, Brain Injury Rehabilitation Trust, Burgess Hill, West Sussex

Robin Morris, Department of Psychology, Institute of Psychiatry, De Crespigny Park, London Huw Williams, School of Psychology, University of Exeter, Exeter Sydney Committee

Robyn Tate, (Convenor), Rehabilitation Studies Unit, Faculty of Medicine, University of Sydney and Royal Rehabilitation Centre, Sydney

Wendy Longley, Multiple Sclerosis Society of New South Wales, Lidcombe

Agnes Rappaport, Commonwealth Rehabilitation Service, Darlinghurst Unit, Sydney

SESSION 1: SERVICES AND PROCESSES

\section{Opening Address: Community-based Rehabilitation Following Traumatic Brain Injury}

Jennie Ponsford

Department of Psychology, Monash University, and Monash-Epworth Rehabilitation Research Centre, Melbourne, Australia

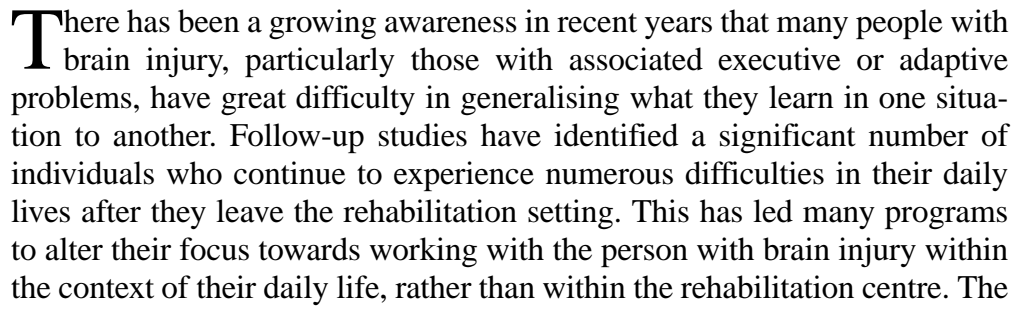


development of a community-based rehabilitation program will be described. This program, and the process of goal setting and measurement and attainment of goals, focuses on the roles and tasks relevant to the injured person in real work contexts, with active involvement of the injured person and close others. Results of a study evaluating a 2-year outcome following this community-based intervention, in a group of 77 individuals with severe traumatic brain injury and 77 matched controls who received centre-based care will be presented, demonstrating the strengths and weaknesses of this model of intervention, and highlighting the importance of contact with trained therapists. Important issues in the implementation of such community-based programs will be discussed.

\section{Working Alliance and Patient Compliance in Brain Injury Rehabilitation and Their Relation to Psychosocial Outcome}

Michael Schönberger ${ }^{1,2}$, Frank Humle ${ }^{1}$, Peter Zeeman ${ }^{1}$ and Thomas W. Teasdale ${ }^{2}$

${ }^{1}$ Center for Rehabilitation of Brain Injury, University of Copenhagen, Denmark

${ }^{2}$ Department of Psychology, University of Copenhagen, Denmark

G mployment and physical activity at follow-up of 98 patients who Cunderwent a holistic neuropsychological outpatient rehabilitation were related to therapeutic process factors. Patients had had a traumatic brain injury $(n=26)$, a cerebrovascular accident $(n=58)$ or another neurological insult $(n=14)$. Two staff members, a neuropsychologist and a physiotherapist, retrospectively rated patients' compliance with the therapeutic regime and their working alliances. Both raters filled out the rating forms separately but had, to some degree, common knowledge about the patients. While the compliance ratings were closely associated, working alliance ratings differed between the raters. The working alliance ratings were predictive of employment, but not physical activity. Both compliance ratings predicted physical training, but only the neuropsychologist's compliance rating was associated with follow-up employment. Post hoc analysis showed that also high compliance ratings given by the physiotherapist were a predictor of employment. Overall, there was a tendency for the neuropsychologist's ratings to be closer associated with employment than the physiotherapist's ratings. Although both raters agreed significantly on their process ratings, results indicate that employment and physical activity are differentially predictable from different process measures rated from different professional perspectives.

\section{The Implementation of a Neuropsychological Rehabilitation Centre in a Private General Hospital in Brazil}

Anita Taub

Psychology and Neuropsychology Division, Hospital Israelita Albert Einstein, Brazil

Tn Brazil, neuropsychology is in its inception and is a new field of knowlledge. Neuropsychological rehabilitation is even less widespread, with currently two reference centres, a public hospital and Hospital Israelita Albert Einstein (HIAE). With 426 beds, HIAE is a private general hospital specialising in highly complex cases; one of its strategic areas is research and treatment of diseases of the central nervous system, and in particular, acquired brain injury. These characteristics led to the creation of a neuropsychological rehabilitation service based on the assumption 
that rehabilitation in its latu sensu starts when a patient victim of a brain injury is admitted to hospital. By means of a partnership with the Oliver Zangwill Centre in England, our model for neuropsychological rehabilitation assistance was set up, focusing on comprehensive care of the patient from the acute phase to social reintegration through individual and group therapy. The main challenge of neuropsychological rehabilitation in our environment relates to calling the attention of victims, health professionals and medical/health insurers to the relevance of this kind of therapy for effective social reintegration of the patient and the development of programs of vocational rehabilitation.

\title{
SESSION 2: MEMORY REHABILITATION
}

\section{Biological Approaches to Memory Rehabilitation After Acquired Brain Injury}

Jonathan Evans

Department of Psychological Medicine, Gartnavel Royal Hospital, Glasgow, UK

\begin{abstract}
Conventional wisdom suggests that restorative approaches to memory rehabilitation are ineffective and hence compensatory approaches form the mainstay of neuropsychological rehabilitation interventions for memory impairment after brain injury. However, recent developments in stem cell research and neuropharmacology suggest that in the future, biologically based restorative treatments may have a place in memory rehabilitation. This paper will review recent developments in biologically based treatments for cognitive dysfunction, and discuss their application after acquired brain injury. Two strands of research will be considered. Stem cell-based brain repair approaches have shown some promise in animal models and in some clinical groups. Issues concerning the application of this methodology after acquired brain injury will be considered. Neuropharmacological interventions using acetylcholinesterase inhibitors are now standard practice in the treatment of dementia of the Alzheimer's type. The limited applications of this approach in people with traumatic brain injury will be reviewed. It is concluded that, for the time being, compensatory approaches to memory rehabilitation are the interventions of choice after acquired brain injury, but biological interventions are an important priority for further evaluation.
\end{abstract}

\section{Memojog - Successes and Limitations of a Memory Aid System}

Andrea Szymkowiak', Kenny Morrison², Peter Gregor², Prveen Shah ${ }^{\text {, Jonathan Evans }}{ }^{4}$ and Barbara Wilson ${ }^{3}$

${ }^{7}$ Division of Psychology, School of Social and Health Sciences, University of Abertay Dundee, Dundee, Tayside, UK

${ }^{2}$ Applied Computing, University of Dundee, Dundee, Tayside, UK

${ }^{3}$ Oliver Zangwill Centre, Princess of Wales Hospital, Ely, UK

${ }^{4}$ Department of Psychological Medicine, Gartnavel Royal Hospital, Glasgow, UK

$\mathrm{M}$ emory problems are among the most common effects of brain injury and can also occur with the ageing process. Prospective memory problems are characterised by the inability to remember to execute tasks at certain times in the future, such as preparing breakfast. Electronic memory aids have been used successfully to give short reminders to individuals with prospective memory problems, usually in visual or auditory 
form. This paper discusses the implementation of a memory aid system we have developed and evaluated with memory-impaired participants. More specifically, we focus on the pitfalls and lessons learned in the process of the development of this system, from the first stages of functional analysis and requirements gathering involving participants to the stage of data gathering and analysis. Methodological and technical challenges are identified and their relevance discussed in the context of system development. Though the evaluation data of the memory aid are promising, much has been learned which will enable us to address limitations of the present system and to develop a more useful and usable system. As the technology for the design of electronic memory aids becomes ever more powerful and versatile, the applications of such systems within assistive technology seem to be too great an opportunity to be missed. Some of these will be discussed.

\section{Prospective Memory in Multiple Sclerosis}

Jennifer Foley ${ }^{1}$, Barbara Wilson ${ }^{1}$ and Agnes Shiel ${ }^{2}$

${ }^{1}$ Medical Research Council, Cognition and Brain Sciences Unit, Cambridge, UK

2 Faculty of Medicine and Health Sciences, NUIGalway, Ireland

Z xamples of prospective memory include remembering to make a - phone call at a certain time or to take medication in the morning. Prospective memory, or "realising delayed intentions" is one of the most commonly used types of memory and its impairment significantly disabling. Several authors have found evidence to suggest that this type of memory is impaired in individuals with Multiple Sclerosis (MS). These studies used individualised assessment measures specific to their studies, however, rather than using standardised methodology. Using the newly developed Cambridge Prospective Memory Test (CAMPROMPT) and other tests of neuropsychological and psychosocial functioning, participants with MS, as compared to healthy controls, were shown to have comparative deficits in prospective memory. These were significantly correlated with some other neuropsychological measures, including those assessing memory. Performance on the CAMPROMPT was also significantly correlated with Barthel Index, EDSS and disease course, suggesting that prospective memory is mediated by MS disease type, impairment and resulting disability. No significant relationship was found to exist with the attentional and executive functioning measures. However, this may have been due to the very low number of individuals who were able to participate on these tests. As expected, performance was not found to be statistically significantly correlated to fatigue, locus of control, anxiety or depression. The results indicate that the CAMPROMPT is a sensitive measure of prospective memory in MS.

\section{The Effects of Reminders on Autobiographical Memory Following Traumatic Brain Injury}

Skye McDonald and Valerie Rendle

School of Psychology, University of New South Wales, Sydney, Australia

$\mathrm{T}$ o date, the rehabilitation of autobiographical memory has been relatively neglected, despite the importance reminiscing plays in normal social relations. There is, however, an experimental literature that focuses 
upon the effects of reminders to facilitate memory for events in both the elderly and the very young. This literature has direct relevance to people with poor memory following traumatic brain injuries (TBI). We report here a study, based on one used with elderly adults, to examine the effect of photographic partial reminders on recall of incidentally learned complex events for 20 adults with TBI. All participants were shown videotapes of some people engaged in everyday activities. Two days later, 10 participants were shown photographic reminders of half the events in the videos shown on three separate occasions. The other 10 participants were given control tasks. All 20 participants were then tested for free recall and recognition of the videotaped events. The "review" group recalled a greater percentage of reviewed events and objects per events recalled, compared to the control group. Photographic review did not impede recall of non-reviewed events. Nor did it affect the recognition of reviewed or non-reviewed events, suggesting that review improved retrieval rather than encoding/storage. While not statistically significant, there were qualitative indications to suggest that "review" participants recalled details that were not available from the photographic review. This suggests that review improves recall of the events themselves rather than the photographs alone. These results suggest that photographic or other review may facilitate reminiscing after TBI.

\title{
Using Errorless Learning in Memory Rehabilitation: Making the Most of the Technique
}

\author{
Catherine Haslam¹, Rebecca Tailby² and Donna Gilroy' \\ ${ }^{1}$ School of Psychology, University of Exeter, Exeter, UK \\ 2 Department of Psychology, The Australian National University, Australia
}

Grorless learning (EL), based on prevention of errors during learning,

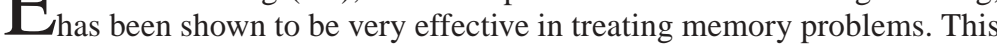
is evident in results of individual (e.g., Clare et al., 1999) and group studies (Baddeley \& Wilson, 1994; Squires et al., 1997) involving patients suffering from a variety of conditions such amnesia and Alzheimer's Disease. While the value of EL is not in doubt, questions remain over the conditions under which we can produce the best results possible. We addressed this general question in two studies. In the first, the influence of active participation during learning was examined. When participants were required to generate their own responses under EL conditions performance was significantly better than that under standard examiner-generated conditions. A second study investigated partial learning to determine whether EL maintained its benefits when knowledge across the entire range, from general to increasingly more specific, was considered. Participants were asked to learn face-name-occupation associations and memory was tested by asking a series of questions tapping knowledge at these various levels. The findings suggest that EL may be no better than trial and error learning when attempting to show partial knowledge (e.g., discriminating studied from non-studied people), but maintains its advantage when more specific detail is sought (e.g., memory for specific occupation or name). Together, these results indicate that there are conditions under which EL can be more effective and suggest that these factors should be taken into consideration when targeting treatment. 


\title{
SESSION 3: CLINICAL ISSUES IN REHABILITATION
}

\section{Approaches to Assessment and Rehabilitation of People in States of Reduced Awareness}

Ages Shiel ${ }^{1}$, Barbara A. Wilson², L.E. Elliott ${ }^{3}$, Jennifer Foley², D. Menon ${ }^{4}$

and J.D. Pickard ${ }^{5}$

${ }^{1}$ Faculty of Medicine and Health Sciences, NUIGalway, Ireland

${ }^{2}$ Medical Research Council Cognition and Brain Sciences Unit, Cambridge, UK

${ }^{3}$ Department of Physiotherapy, Addenbrookes Hospital, Cambridge, UK

${ }^{4}$ Department of Anaesthesia, University of Cambridge, UK

${ }^{5}$ Department of Academic Neurosurgery, University of Cambridge, UK

\begin{abstract}
A dmission to many rehabilitation units is dependent on the patient's ability to engage and participate in rehabilitation programs. Yet, this may exclude those patients who are slow to recover full consciousness and/or remain in states of reduced awareness. In addition, even for those who recover more rapidly, the window of opportunity to address impairments caused by brain injury may be reduced. Rehabilitation after brain injury should begin immediately. Even patients in the very early stages of recovery, for example, those who are still dependent on ventilators benefit. Accurate and regular assessment is central to provision of appropriate intervention. If appropriate assessment is not carried out, it may appear that progress has ceased, whereas subtle gains are being made over weeks, months or even years. Assessment should also be carried out at different times of day and in different environments, as meaningful behaviours may occur at infrequent and unpredictable intervals and responses are rarely if ever consistent. When such gains and/or behaviours are correctly identified they can be consolidated with some patients when treated in an appropriate environment by a multidisciplinary team. Approaches to rehabilitation of people in states of reduced awareness may be restorative, compensatory or adaptive. Regardless of the approach, many of the techniques and methods employed are similar. These include provision of a stimulating environment, the interaction of people in the environment with the patient and postural changes. A series of single case studies including coma, the vegetative state and the minimally conscious state will be used to illustrate how appropriate assessment and treatment methods can be applied regardless of location.
\end{abstract}

\section{Assessment of Fitness to Drive in People with Dementia}

Nadina B. Lincoln, Elizabeth Lee and Alice Reay

School of Psychology, University of Nottingham and Walton Hospital, Chesterfield, UK.

P revious studies have suggested that cognitive tests could be used to advise people with dementia when they should give up driving. However specific criteria have often not been validated in comparison with "on road" assessment. Healthy elderly people and people with dementia were assessed on measures of memory, concentration, reasoning and perception. They were assessed on the road by a driving instructor blind to the cognitive test results. Participants were graded as definitely safe, probably safe, probably unsafe and definitely unsafe to drive. All 32 healthy elderly drivers were judged safe to drive. Ten of the 37 drivers with dementia were found to be unsafe. There were significant differences between healthy elderly and patients with dementia on the cognitive tests. There were no 
significant differences between safe and unsafe drivers with dementia on individual cognitive tests. Discriminant function analysis indicated that $97 \%$ of participants with dementia could be correctly classified as safe or unsafe to drive on the basis of performance on a combination of 7 cognitive tests. Performance on individual cognitive tests was not associated with safety to drive in people with dementia. However, a combination of measures was able to differentiate those found safe to drive from those who were unsafe. Prospective testing of the predictive equation derived with this sample is needed.

\section{Capacity to Consent to Rehabilitation: Assessment in Clinical Practice}

Camilla Herbert

Brain Injury Rehabilitation Trust, Burgess Hill, West Sussex, UK

New legislation in the United Kingdom (England and Wales) is being 1 proposed that will significantly alter the assessment of mental incapacity. There is already new legislation in Scotland (Incapacity Act Scotland 2000). Ethical issues in the assessment of capacity to consent to treatment will be discussed in relation to the draft legislation. The assessment of capacity to consent to rehabilitation rather than specific medical interventions will be discussed, and the complex issues arising from longer term support packages in the community will also be addressed. Although legal jurisdictions approach the issues surrounding capacity in different ways, the issues to be addressed in clinical practice are universal. Using clinical examples, this paper will explore the theoretical approaches to determining capacity, the abilities commonly assessed in determining capacity, and the principles underlying healthcare decision making for adults who lack capacity. It will focus particularly on the competing demands of the individual's right to self-determination and requirement to consider the duty of care by the professional or service provider.

\section{Do Neuropsychologists in Rehabilitation Make the Most of their Assessment Data?}

Wendy A Longley ${ }^{1}$ and Robyn L Tate ${ }^{2}$

${ }^{1}$ Multiple Sclerosis Society of New South Wales, Lidcombe, Australia

${ }^{2}$ Rehabilitation Studies Unit, Faculty of Medicine, University of Sydney and Royal Rehabilitation Centre Sydney, Australia

The predominant role of clinical neuropsychologists in Australian neu1 rological rehabilitation units is diagnostic. This is largely a function of the focus of most neuropsychological educational programs in which the almost exclusive focus of training is on the assessment process, with scant attention paid to the remediation of those impairments identified in the neuropsychological examination. In many instances, a single feedback session and, possibly, a copy of the report from the assessment is the only post-assessment contact a neuropsychologist has with the client (Gordon \& Muenchberger, 2001; Muenchberger \& Gordon, 2001; Longley, Turner, Naismith et al., in preparation). This model of practice continues to be valued in Australia by employers and clinicians alike. Yet there is little scientific evidence of its worth, and what evidence does exist calls into question that perceived value. In one study, a single feedback session from the neuropsychological assessment was shown not to be effective 
(Beardmore et al., 1999). Another study (Bennett-Levy et al., 1994) found that clients rated a significant proportion of feedback as either not useful or not understandable. So, there is some uncertainty about whether this heavy investment into gathering such rich diagnostic assessment data is fully reaped in terms of actual benefit to the client undergoing rehabilitation. In the present paper, the authors outline current problem areas in the translation of assessment data into benefit for the client undergoing rehabilitation and present a method for evaluating the current practice model. The paper concludes with the proposal of an alternative "neuropsychoeducation focused" model for clinical practice that should ensure more reliable and effective translation of assessment data into client benefit.

\title{
Hypoxic Brain Damage: Individual Differences, Compensatory Strategies and Vocational Outcome
}

\author{
Agnes Rappaport, Donna Wakim and Michelle Dooley \\ Commonwealth Rehabilitation Service, Darlinghurst Unit, Sydney, Australia
}

\begin{abstract}
$\mathrm{R}_{\mathrm{s}}$ eturn to independence and competitive employment following acquired brain impairment (ABI) can be a slow and difficult process. This presentation discusses vocational outcomes following two cases of hypoxic brain damage, taking into account not only cognitive deficits, but individual differences and readiness to use compensatory strategies. The first case involves a 44-year-old woman who had memory and new learning deficits, slowness in information processing and difficulty with executive functions. There was lack of initiative and decreased confidence. Despite these deficits, she passed her driving test 7 months later, and returned to work as a lecturer, on a part-time basis. The second case concerns a 24-year-old male whose hypoxic brain damage resulted in marked memory and new learning deficits, slowness in information processing and deficits with executive functions. He passed his driving test 13 months later; however, return to part-time university study was not successful. He commenced a program with Commonwealth Rehabilitation Service (CRS) in June, 2003 and suitable vocational goals are still being explored. At the initial stages of rehabilitation, both were disabled by their ABI, although the second case had more severe deficits. Compensatory strategies were more beneficial in the first case. In the second case, the process of working through different vocational options with his CRS case manager and ongoing professional support is slowly bringing about increased awareness. These two cases highlight the importance of taking into account individual differences in relation to the use of compensatory strategies, speed of recovery and levels of support needed.
\end{abstract}

\section{SESSION 4: EVIDENCE-BASED PRACTICE}

\section{Treatment of Cognitive Problems in People with Multiple Sclerosis}

\author{
Nadina B. Lincoln
}

School of Psychology, University of Nottingham, Nottingham, UK.

Cognitive problems in Multiple Sclerosis (MS) are common and may
require treatment. The aim of the study was to evaluate the effectiveness
of cognitive rehabilitation in individuals with MS. Patients were recruited as
part of a randomised controlled trial evaluating cognitive assessment and 
intervention in the management of MS. Treatment in individuals was evaluated using ABA single-case experimental designs. Patients completed diaries to monitor the effects of intervention. Intervention aimed to reduce the practical impact of cognitive impairment on daily life. Of the 79 patients allocated to treatment, $36(46 \%)$ received it and $29(37 \%)$ completed the intervention. Comparing baseline and intervention diaries showed that 20 of $29(69 \%)$ patients appeared to benefit from treatment. There was a significant reduction in the occurrence of cognitive problems in daily life following the intervention $(p<.01)$. There was no significant change in mood or quality of life. Factors influencing the uptake of a cognitive rehabilitation program and affecting whether patients benefited from the intervention will be discussed. Cognitive rehabilitation reduced daily life problems for some patients with MS and further evaluation of specific cognitive deficits that benefit from intervention is warranted.

\section{Knowledge and Utilisation of Evidence-based Clinical Practice in Rehabilitation of Acquired Brain Impairment: The Timeliness of PsycBITE ${ }^{\mathrm{TM}}$}

Michael Perdices ${ }^{1}$, Robyn Tate ${ }^{2}$, Skye McDonald ${ }^{3}$, Leanne Togher ${ }^{4}$, Anne Moseley², Kiri Winders ${ }^{2}$, Regina Schultz ${ }^{2}$, Kate Smith ${ }^{2}$ and Maria Kangas ${ }^{5}$

${ }^{1}$ Department of Neurology, Royal North Shore Hospital, Sydney, Australia

${ }^{2}$ Rehabilitation Studies Unit, Faculty of Medicine, University of Sydney and Royal Rehabilitation Centre Sydney, Australia.

${ }^{3}$ School of Psychology, University of New South Wales, Sydney, Australia

${ }^{4}$ Department of Communication Sciences and Disorders, Faculty of Health Sciences, University of Sydney, Sydney, Australia

${ }^{5}$ School of Psychology, Macquarie University, Sydney, Australia

$\mathrm{T}$ This survey documented work practices of, knowledge of, and attitudes towards utilising evidence based clinical practice (EBCP) among practitioners working in acquired brain injury rehabilitation in New South Wales, Australia. Respondents were 63 relatively experienced practitioners. Most were psychologists (35\%), although a wide range of allied health professionals was represented. Most respondents (69\%) worked with a varied clinical population, most commonly traumatic brain injury $(89 \%)$ and stroke (56\%). Practitioners treated a range of problem areas and used a wide repertoire of interventions. The majority of respondents $(53 \%)$ reported having considerable experience with their most recently treated problem. Reliance on their past experience or consultation with colleagues were the most common strategies used to guide the choice of intervention (43\%). Only $29 \%$ consulted the literature to help determine their choice of intervention. Although all respondents reported evaluating their intervention in some manner, only $10 \%$ used pre- and posttreatment measures to evaluate the effect of therapy. Whether or not this method was used was unrelated to the practitioner's years of experience. Only $38 \%$ thought that EBCP was important or crucial and $4 \%$ reported exclusive adherence to EBCP in their work. Length of clinical experience was unrelated to respondents' perception of the importance of EBCP or the proportion of time they adhered to EBCP in their clinical work. These data suggest that reliance on EBCP is poor and highlight the need for an appropriate resource, such as PsycBITE ${ }^{\mathrm{TM}}$, to increase awareness and practice of these principles. 


\section{PsycBITE ${ }^{\mathrm{TM}}$ : An Internet Resource for Evidence-based Treatment of Acquired Brain Impairment}

Robyn Tate ${ }^{1}$, Michael Perdices², Skye McDonald ${ }^{3}$, Leanne Togher ${ }^{4}$, Anne Moseley', Kiri Winders ${ }^{1}$, Maria Kangas ${ }^{5}$, Regina Schultz ${ }^{1}$ and Kate Smith ${ }^{1}$

${ }^{1}$ Rehabilitation Studies Unit, Faculty of Medicine, University of Sydney and Royal Rehabilitation Centre Sydney, Australia

2 Department of Neurology, Royal North Shore Hospital, Sydney, Australia

${ }^{3}$ School of Psychology, University of New South Wales, Sydney, Australia

${ }^{4}$ Department of Communication Sciences and Disorders, Faculty of Health Sciences, University of Sydney, Sydney, Australia

${ }^{5}$ School of Psychology, Macquarie University, Sydney, Australia

The first part of this paper describes the developmental work underpinning a unique database resource containing published reports of therapies for the psychological consequences of acquired brain impairment (ABI). The resource, entitled the Psychological database for Brain Impairment Treatment Efficacy (PsycBITE ${ }^{\mathrm{TM}}$ ), was primarily developed to aid clinicians to evaluate and implement evidence-based interventions for individuals with ABI. Reports meeting five selection criteria are eligible for inclusion on PsycBITE ${ }^{\mathrm{TM}}$ : the population (i) has brain impairment of acquired aetiology, (ii) is older than 5 years of age, (iii) receives an intervention that is either psychologically-based or targets a psychological consequence of $\mathrm{ABI}$, and the report (iv) is a full-length publication in a peer-reviewed scientific journal and (v) provides empirical data on treatment outcomes. Seven databases are searched, using 85 terms. Reports are indexed using 72 terms, enabling users to specify selection of reports in any of the following domains: problem area, type of intervention, neurological group, age group and study design. A distinctive feature of PsycBITETM is the rating of the methodological quality of the reports, which are then ranked on the database in order of their methodological strength. The second half of the presentation will include an on-line demonstration of searching the database.

\section{Keynote Address: The Clinical Neuropsychologist's Dilemma}

\section{Barbara A. Wilson \\ Medical Research Council - Cognition and Brain Sciences Unit, Cambridge and The Oliver Zangwill Centre, Ely, UK}

This paper suggests that problems faced by therapists, patients and their families involved in cognitive rehabilitation are so diverse and complicated that claims that cognitive rehabilitation itself can be rendered effective by following directions indicated by and emanating from research in the basic cognitive neurosciences cannot be justified. Although Robertson's (2003) elegant and well researched paper entitled "Cognitive Neuroscience and Brain Rehabilitation: A Promise Kept" suggests the contrary, drawing support from studies in plasticity, unilateral neglect in stroke patients, the reduction of attentional deficits in people with brain injury and others, it is the contention of this paper that such research on its own cannot provide sufficient guidelines or a strong enough framework to cope with the scale and complexity of concerns faced by the parties involved in the process of cognitive rehabilitation. A typical scenario is presented in which a young man who has sustained a traumatic brain injury is admitted for rehabilitation with a number of problems, for example, memory, attention, planning and organisational difficulties. He has emotional difficulties, such as anxiety, depression and mood 
swings; he has behavioural problems, including poor self-control and verbal aggression. His family needs help, and the young man himself wants to return to work. Most of these problems are not the focus of research in the neurosciences. Obviously, we can call upon research from the neurosciences to help in reducing specific difficulties. We might be able to arrange a PET scan or functional MRI to help obtain guidance as to whether to use a restitution or compensatory strategy. However, such contributions are at best limited in the face of the multiplicity of concerns that face the participants in cognitive rehabilitation. It is suggested that a much broader theoretical framework is needed, which should include models of cognition, emotion, behaviour, recovery, together with empathy and clinical skills.

\title{
5: ACTIVE POSTER SESSION
}

\section{Clinical Implications Resulting from a Study to Develop an Ecological Test of Divided Attention}

Eve Greenfield ${ }^{1}$, Jonathan Evans ${ }^{2}$ and Barbara Wilson 1

${ }^{7}$ Medical Research Council - Cognition and Brain Sciences Unit, Cambridge, UK

${ }^{2}$ Section of Psychological Medicine, Division of Community Based Sciences Academic Centre, Gartnavel Royal Hospital, Glasgow, UK

\begin{abstract}
fter acquired brain injury many people complain that they find it difficult A to do more than one thing at a time. This has serious implications for rehabilitation and everyday life. We have developed a new, ecologically based test battery to assess divided attention and produced a tool to accurately measure the decrement between performance of single and dual tasks. Both cognitive and motor tasks are included in the new test. Our results have important implications for the assessment, treatment and management of problems of divided attention. In this paper we provide clinical examples to illustrate some of the interesting findings in these areas that emerged during the study. As predicted, there was a significant difference in the decrement between the patient and control groups in all subtests. However, there were also differences in resource allocation between the groups, that is, which task was prioritised or sacrificed. We also demonstrated that even those patients who could carry out two tasks simultaneously found it more difficult to sustain their attention in tests involving the more demanding cognitive task. We looked at differences in performance between the different diagnostic groups, as well as the effects of age, gender and IQ. Some useful treatment ideas and strategies emerged, for example, many found that engagement in a repetitive motor task facilitated their concentration on a demanding cognitive task. These findings can be directly transferred to rehabilitation and everyday situations, to enable people to manage impairments in divided attention more successfully.
\end{abstract}

\section{Developmental Coordination Disorder: A Virtual Reality Study Proposing the Facilitation of Focused Rehabilitation by Distinguishing Precise Underlying Deficits}

Ros Watling and Ashok Jansari

School of Psychology, University of East London, London, UK

Developmental coordination disorder (DCD) is a condition involving a marked impairment in the development of motor coordination which impacts on learning and activities of daily living. As well as difficulties with fine and gross motor skills there may be difficulties with ocular-motor 
function, planning and organisational skills, language and speech. The term DCD is used loosely to describe "praxis" difficulties; however, these difficulties may be due to one or more underlying deficits including perceptual processing, kinaesthetic perception and motor execution of planned actions. An important issue for both research and rehabilitation is understanding the contribution of these different factors to an individual's difficulties. A study is reported which aims to distinguish perceptual deficits from motor deficits using a novel virtual environment (VR) paradigm. Participants are tested for manual dexterity using a pegboard that requires both perceptual and motor abilities. A second test is administered using a computerised VR replication of the pegboard in which the motor component is greatly reduced because the user moves the pegs with a mouse, thus eliminating the tactile and grip force components of the movements. Finally, tests of pure manual dexterity and perceptual ability are administered to partial out their individual contributions. It is hoped that such a paradigm will enable a distinction between perceptual and motor deficits in DCD, thus facilitating a more precise understanding of the individual's movement problems. Such insight would allow specifically tailored support and intervention at a rehabilitative level, as well as increased subjective awareness and understanding for the sufferer.

\title{
Effort Testing Can Have a Greater Effect on Neuropsychological Testing Outcome Scores than Severe Brain Injury
}

\author{
Stephanie Moore \\ Department of Neurology, University of California, Irvine, USA
}

$\mathrm{N}$ europsychologists can no longer overlook the profound influence effort plays on neuropsychological test profiles in adults and children. Subjects who apply insufficient effort on very simple tests, such as the Word Memory Test and the Computerised Assessment of Response Bias, can distort the outcome of their neuropsychological profile to a substantial degree. For instance, when comparing these patients to those with severe brain injury, those with the most impairment score the highest on effort tests. Although most research studies find the largest effect of effort on tests of memory, many other tests are also influenced by effort. Given that the number of patients failing effort tests is not negligible, effort must be measured to insure a valid profile, not only with private patients but also in research studies. Comparisons will be made among a variety of diagnostic groups, such as chronic fatigue, pain, depression, and head injuries, to demonstrate the importance of determining genuine from exaggerated impairment.

\section{Evaluation of Subjective Functioning in Spinal Cord Injured Patients Using the Ruff Neurobehavioral Inventory}

Robin Murray ${ }^{1}$, Ali Asghari', Dmitri Egerov${ }^{1}$, Michael Nicholas', Sue Rutkowski', Phillip Siddall ${ }^{1}$ and Ronald Ruff ${ }^{2}$

1 University of Sydney Pain Management and Research Centre, Royal North Shore Hospital, Sydney, Australia

2 Department of Psychiatry, University of California, San Francisco, USA

The Ruff Neurobehavioral Inventory (RNBI) was developed to fulfil a perceived lack in neuropsychology rehabilitation: an instrument that would measure the most important aspects of life activities for those whose 
lives have been affected by a catastrophic event such as an illness or injury. The RNBI measures the individual's subjective assessment of their functioning pre- and postevent (Ruff \& Hibbard, 2003). To assess the clinical usefulness of the RNBI with an Australian sample of spinal cord injured patients, 33 patients were assessed. Overall, responses produced valid profiles. A statistically significant difference was found between pre and postmorbid cognitive functioning, and on the four subscales that make up the composite cognitive measure: attention and concentration; executive functions; learning and memory; and speech and language. This finding suggested an unexpected subjective lowering of cognitive abilities. Statistically significant differences were expected and found between pre and postmorbid functioning on physical and quality of life composite scores and on several of the subscales that make up these two scales. No significant difference emerged between pre and postmorbid emotional composite scales. Findings suggest that the RNBI is clinically useful when spinal cord injured patients are assessed for rehabilitation. Given this group's subjective lowering of cognitive abilities, and the possibility of head trauma during injury, neuropsychological screening following spinal cord injury is recommended.

\section{Translating Research Findings for Parents of Children with Spina Bifida and Hydrocephalus to Aid Rehabilitation}

Arleta Starza-Smith and Hazel Bennett

Department of Clinical Psychology and Neuropsychology, University Hospital Nottingham, Nottingham, UK

T ong-term rehabilitation is almost a way of life for many people affected by spina bifida and hydrocephalus throughout childhood and adolescence, including coping with evolving problems, repeated hospital admissions and remissions. The Association for Spina Bifida and Hydrocephalus (ASBAH) is committed to providing support to members, often through commissioning research. The investment of charitable funds in research must result in effective dissemination of findings to benefit the association's membership. This paper discusses ASBAH's undertaking to present recently commissioned research in an accessible format to members, drawing on data on 116 children and adolescents, and focussing on the process of interpreting findings into a format which will facilitate extrapolation of information and accelerate the process of rehabilitation through active involvement of the client and their family. The involvement as a "translator" of the clinical neuropsychologist with hands-on clinical experience is paramount in creating the essential theory-practice "link" between the research team's findings and the practical application in service delivery to ASBAH membership, resulting in an atmosphere of "patient partnership" in rehabilitation. The current paper will outline findings relating to cognitive, social, emotional and educational outcomes in two age cohorts of 6-12 years and 13-18 years and discuss the challenges faced by ASBAH's committee members in bringing crucial research information to its membership. Case examples will be used to illustrate pathways with focus placed on the transitional adolescence years. 


\title{
Behavioural Assessment of the Dysexecutive Syndrome for Children (BADS-C): Utility in Clinical Practice?
}

\author{
F. Colin Wilson ${ }^{1}$, Hazel Emslie ${ }^{2}$, Vivien Burden³, Ian Nimmo-Smith² \\ and Barbara A. Wilson² \\ ${ }^{1}$ Green Park HealthCare Trust, Joss Cardwell Centre, Belfast, UK \\ ${ }^{2}$ Medical Research Centre - Cognition and Brain Sciences Unit, Cambridge UK \\ ${ }^{3}$ Private Practice, Cambridge, UK
}

\begin{abstract}
$\mathrm{N}$ umerous studies of broad intellectual functioning in children and adolescents exist, however fewer studies have investigated higher order "executive" functioning. Despite numerous attempts to delineate executive functioning in adults, assessment and quantification has proven problematic (Crawford, 1998). Undoubtedly quantifying the set of competencies which define "executive functioning", such as ability to plan, sequence, sustain attention to task, resist distraction, manage feedback, coordinate activity and change response set is even more challenging in the context of an evolving cognitive system, although evidence exists to support the concept of a developmental hierarchy of executive skills from simple planning, hypothesis testing and impulse control through to efficient complex planning (Levin et al., 1991). Unfortunately most traditional measures are insensitive to executive dysfunction (Anderson, 1998). Nevertheless the effects of executive dysfunction arising from impaired neurodevelopment or trauma should become more evident with age as the child is exposed to increasingly complex cognitive, educational and social demands. The BADS-C was adapted from the Adult BADS (Wilson et al., 1996) following pilot studies in an attempt to define executive level developmental competencies. Data from control $(n=259)$ and clinical $(n=114)$ participants aged $8-15$ will be presented. Children with attention deficit hyperactivity disorder, PDD and traumatic brain injury differed significantly from controls and those with dyslexia. Self-report (DEX-C) and Goodman's Strengths and Difficulties questionnaire data from teachers and parents was significantly negatively correlated with BADS-C performance. Correlations between measures of verbal fluency, memory, attention and BADS-C indicated that BADS-C is examining independent developmentally appropriate cognitive domains, which may assist in clinical decisionmaking and formulation.
\end{abstract}

\section{SESSION 6: TECHNOLOGIES AND VIRTUAL REALITY}

\section{Virtual Reality Investigation of Strategy Formation, Rule Breaking, Prospective Memory, and Hazard Decision-making in Patients with Closed Head Injury}

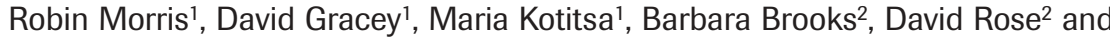
Simon Fleminger ${ }^{3}$

${ }^{1}$ Neuropsychology Unit, Institute of Psychiatry, De Crespigny Park, London, UK

2 Department of Psychology, University of East London, UK

${ }^{3}$ The Lishman Unit, Maudsley Hospital, London, UK

$\mathrm{V}$ irtual reality (VR) technology can be used to display complex visual images that change rapidly in response to instructions from users. In this fashion it has the potential to provide a more ecologically valid approach to neuropsychological assessment. However, it needs to be 
established as to whether or not VR assessment procedures are sufficiently sensitive and specific enough to detect neuropsychological deficits across a range of patient types. The present study sought to make use of VR technology to assess executive functioning in patients who had endured a closed head injuries (CHI). Three VR tasks were used for this study, which compared $20 \mathrm{CHI}$ patients with 20 matched normal controls: (1) A Bungalow Task assessed strategy formation, rule breaking and prospective memory; (2) a Warehouse Task was used to test strategy formation, rule breaking and an ability to efficiently allocate resources across tasks; and (3) a Kitchen Task measured decisionmaking abilities regarding hazard detection and evaluation in a domestic environment. It was shown that CHI patients showed specific deficits in strategy formation, rule breaking and time-based prospective memory, but not event-based prospective memory or decision making. These tasks proved to be more sensitive to impairment than convention "ecologically" valid psychometric tests. The findings support the use of VR to investigate executive dysfunction in $\mathrm{CHI}$ and suggest that this approach can be used to bridge the gap between laboratory-based experimental procedures and "ecologically" valid approaches.

\title{
The Use of Virtual Reality to Assess and Predict Real-world Executive Dysfunction: Can VR Help for Work-placement Rehabilitation?
}

\author{
Ashok Jansari ${ }^{1}$, Robert Agnew ${ }^{1}$, Katarina Akesson ${ }^{1}$ and Lesley Murphy ${ }^{2}$ \\ ${ }^{1}$ School of Psychology, University of East London, UK \\ ${ }^{2}$ RehabUK, London, UK
}

Gocal lesions to the frontal lobes can result in a range of "higher order" problems with tasks involving planning, decision-making, prioritisation, and so forth. Although standard clinical tests such as the Wisconsin Card Sorting Test and Tower of London can capture some of these deficits, their ability to predict "real-world" adjustment has been called into question. For instance, Shallice and Burgess (1991) found that some frontal patients who showed no signs of abnormality on neuropsychological tests, nonetheless were significantly impaired on a naturalistic shopping paradigm. Such findings suggested the need for more ecologically-valid tests being developed with the Behavioural Assessment of the Dysexecutive Syndome (BADS, Wilson et al., 1996) being one particular example. Using this as one of their measures, McGeorge et al. (2001) compared five patients' performance on a task involving an office environment with their performance on the same task using a virtual reality (VR) environment. Surprisingly, they found that whilst the patients performed poorly on both the real and VR tasks, their BADS scores were within normal limits. A study is reported which replicates and extends the McGeorge one in two major ways. Firstly, a larger sample of patients recruited from RehabUK is used and secondly, the task investigates, at a more fine-grained level, the cognitive constructs that patients may be finding particularly difficult, such as decisionmaking, task selection, sequencing and distractability. The ultimate hope is to be able to produce from this a profile-type summary of the patient's abilities/deficits which a lay person or employer would find useful in attempting to successfully place an individual in work. 


\section{Computer-assisted Cognitive Rehabilitation: Current and Future Development}

David Man

Department of Rehabilitation Sciences, The Hong Kong Polytechnic University, Kowloon, Hong Kong SAR

The potential of computer-assisted cognitive rehabilitation (CACR) for 1 people with cognitive decline is increasingly being recognised. The reliability, validity, user acceptance and cost-effectiveness of computer-assisted assessments are well documented and have suggested equivalence with traditional methods. In the present presentation, neuroplasticity, the interaction effect between human and computer and advantages/limitations of CACR for persons with cognitive deficits will be outlined. The global trends of CACR, namely, compact and customisable; internet-based; be intelligent; virtual-reality based; and client-led will be illustrated with examples of application and the Cognitive Rehabilitation Service (CRS) within the Hong Kong Polytechnic University, Hong Kong. The presentation will also highlight the published results of CACR of the author's research team, new focus of functional and social problem-solving in both CACR assessment and intervention. Examples of cultural-relevant, customised software (developed by visual basic, Flash and $\mathrm{C}++$ languages, etc.) will be demonstrated in various aspects: (a) attention training for persons with traumatic brain injury; (b) communication board downloadable/customisable from web-server; (c) Chinese character writing for dyslexic children; (d) use of expert system in memory for professionals, patients and carers; (e) virtual-reality based super-market shopping; (f) tele-assessment using examples of Neurobehavioral Status Examination (or Cognistat) or Rivermead Behavioral Memory Test (RBMT); (g) tele-cognitive rehabilitation for persons with memory (e.g. in recalling object locations within kitchen or bedroom); (h) tele-cognitive rehabilitation for persons with problem-solving difficulties; and (i) the application of Flash Com (a kind of net meeting) to individual and group of 3-4 members (e.g., in grouplearning of memory strategies). Initial findings from some preliminary studies indicated that CACR had significant improvement in memory, problem-solving and functional skills like writing, reading following treatment. CACR's usability and feasibility has been supported by clients' feedback. However, our findings need more support in differentiating efficacy of a CACR and non-CACR programs in different cognitive process such as memory.

\section{Using Video to Help Families and Schools in the Rehabilitation of the Head-injured child}

Thomas P. Kelly, R.J. Forsyth, B. Wicks, and S. Walker

Newcastle Primary Care Trust and Newcastle General Hospital, UK

chool is perhaps the most important source of long-term rehabilitation for $\checkmark$ the child with acquired brain injury. Despite this, families often struggle to have the needs of their head-injured child acknowledged and met within the educational system. Frequently families may not have the resources to advocate for their child, and clinical staff experienced in the management of brain injury are often only available in the acute period of recovery. Providing the school and particularly the child's teachers with information about acquired head injury was considered to be an important step in ensuring adequate 
recognition and provision of need. A short video which parents could give to the teaching staff was considered the most effective tool for conveying information. A video owned by the family could be lent to new teachers and learning support staff at the start of each academic year. Using brief information from educational, psychological and medical staff, as well as interviews with 4 children with acquired brain injury and their families, a 15-minute video was produced. The video provides basic information on the typical cognitive and behavioural problems following acquired brain injury, as well as suggesting appropriate responses. Teachers, children and their families indicate helpful and unhelpful practice.

\title{
SESSION 7: EXECUTIVE FUNCTIONS AND EMOTIONAL PROCESSING
}

\section{Self-knowledge and Self-referential Processing in Memory-impaired Patients}

Elizabeth L. Glisky and Maria J. Marquine

Department of Psychology, University of Arizona, Tucson, USA

\begin{abstract}
$\mathrm{A}$ $\mathrm{n}$ injury to the brain, and its resulting memory deficits, can affect the core of the individual: the self. Although some investigators in the area of personality and social psychology have argued for a loss of sense of self following brain injury, others have distinguished between general knowledge about oneself (semantic self-knowledge) and memory for the events in one's life (episodic self-knowledge). These researchers have proposed that although episodic self-knowledge might be impaired in individuals with a brain injury, semantic self-knowledge is usually intact. Studies in cognitive psychology have demonstrated that engaging in self-referential processing during an encoding task enhances memory; this has been called the self-reference effect (SRE). To our knowledge, the SRE has not been studied in brain-injured patients. The goal of the present study was to assess semantic self-knowledge in memory-impaired individuals through the use of a personality trait questionnaire and to explore the effects of self-referential processing on recognition memory. Preliminary data suggest that engaging in self-referential processing enhances memory performance in patients with moderate memory deficits. Theoretical implications and practical applications of these data will be discussed.
\end{abstract}

\section{Function of the Anterior Cingulate Cortex: Evidence from Three Case Studies}

Bonnie-Kate Dewar ${ }^{1}$, Sergio Zanini ${ }^{2}$, Hugo Critchley ${ }^{3}$, Ray Dolan ${ }^{3}$, Tim Shallice ${ }^{2,4}$ and Lisa Cipolotti ${ }^{1}$

${ }^{1}$ Department of Neuropsychology, National Hospital for Neurology and Neurosurgery, London, UK

${ }^{2}$ Cognitive Neuroscience Sector, International School for Advanced Studies, Trieste, Italy

${ }^{3}$ Wellcome Department of Imaging Neuroscience, Institute of Neurology, London, UK

${ }^{4}$ Institute of Cognitive Science, London, UK

$\mathrm{T}$ The function of the anterior cingulate cortex (ACC) has been recently investigated by neuroimaging studies. This has led to the development of a number of theories of ACC function. One of the best developed accounts is the conflict-monitoring theory (Botvinick et al., 2001). Neuropsychological studies of patients with brain damage allow some of the key theoretical issues underlying these functional theories to be addressed. So far, the majority of the neuropsychological studies investigating the function of the ACC have been rather qualitative. In this paper we 
have systematically investigated the performance of three patients with ACC lesions on a wide series of cognitive tests. A detailed investigation of their performance on clinical and experimental tests of frontal executive function and anterior attention was also undertaken. We found that the patients' performance on focal cognitive tests was essentially normal. Interestingly, the patients also performed remarkably well on frontal executive tasks and two experimental tests of anterior attention. These findings are relevant for current functional theories of the ACC. We conclude that our results are problematic for the conflict-monitoring hypothesis. We support the view that the ACC functions to prospectively bias behaviour through the integration of cognitive processing of uncertainty with adaptive changes in bodily states (Critchley et al., 2001b).

\title{
Decoding Facial Expression: Incidence and Mechanisms of Facial Emotions
}

\author{
Robin Green, Gary Turner and William F. Thompson
}

Toronto Rehabilitation Institute, Toronto, Canada

Gacial emotion perception (FEP) has been little studied in traumatic - brain injury (TBI) despite its important clinical ramifications. Our research program includes the following questions: (1) Is FEP compromised in recent TBI? Previous TBI studies have examined chronic patients. However, differences between recent and chronic (e.g., severity of cognitive impairments, use of compensatory strategies) might differently affect presentation and rehabilitation of FEP impairments. (2) Is diffuse axonal injury (DAI) rather than focal injury, a primary neurological mechanism for FEP impairment in TBI? Adolphs et al. (2000) hypothesised that white matter tract damage might cause FEP deficits. (3) Is FEP impairment a perceptual deficit or secondary to other cognitive deficits? 30 TBI participants and 30 age-matched controls were tested in a $2 \times 3$ mixed design. Dependent variables were accuracy and reaction time on neutral and emotional face perception tests. In relation to aim 1, the TBI group performed significantly less accurately than controls on FEP tasks, whereas the groups performed equivalently on a non-emotional face perception control task, confirming FEP impairment in recent TBI. (2) A subgroup of TBI participants without focal injury to brain areas typically implicated in FEP impairment was as impaired on FEP tasks as a sub-group with focal lesions in these areas, suggesting an alternative neurological mechanism for deficits in the first subgroup, and providing evidence for the DAI hypothesis. (3) Controls allocated extra time to perform the FEP versus control tasks whereas patients did not, and their performance suffered. This suggests that a strategic- or impulse-control deficit contributes to FEP impairment.

\section{SESSION 8: CULTURE AND COMMUNITY}

\section{Culture and Context}

Shirley Tollman ${ }^{1}$

${ }^{1}$ Strathmore Park, Durban, South Africa

Tntegral in the development of the structures underlying thought and action, it is in turn essential for understanding behavioural expression and planning appropriate rehabilitation programs. A valid interpretation of behavioural responses necessitates an understanding of the individual's 
sociocultural context. Thus, a knowledge of the underlying structures that characterise culture and context, in addition to knowledge of brain function and brain-behaviour syndromes is essential for the formulation of an assessment and in turn appropriate planning of an holistic rehabilitation process. "From traumatic head injury to a productive life" is the aim of rehabilitation; reorganisation compatible with the head-injured person's changed capacities and their ideals, to be adapted to their own particular society at large. The rehabilitative team needs to help the head-injured person to find a niche and like it. This paper will focus upon explanations of the world as evolved through the development of different cultures and how these beliefs impact upon behaviour. Cultures contrasted will include "traditional" Westernised beliefs, spirit-based cultures and some Indian profiles. All these cultures are to be found within the rich mosaic of the diverse communities which make up South Africa's rainbow nation. In describing these differences, the shortcomings of an exclusive psychometric approach for capturing behaviour will be pointed to. The areas addressed will include: (1) the relative importance ascribed to individualised versus community based needs; (2) differences of beliefs of past, present and future; of the role of ancestors; of the purpose of the present sojourn in this world and the after-death experience; (3) context spiritual beliefs, to interpret the world in terms of spirits (the influence of history, ancestors and elders); (4) nuclear versus extended family; (5) developmental practices; and (6) attitude towards illness - whether the etiology is internal or external and, in turn, sick role enactment.

\section{Targeted Rehabilitation over a Lifespan}

Helen Harrington ${ }^{1}$

${ }^{1}$ Community Brain Injury Programs, Epworth Rehabilitation, Melbourne, Australia

The field of rehabilitation is growing in its awareness of the long-term 1 issues for a person having suffered a brain injury. A number of research studies highlights the ongoing nature of the cognitive and behavioural deficits often leading the brain-injured person feeling depressed, socially isolated and experiencing difficulty maintaining skills. The informed rehabilitation field is continuing to develop community-based rehabilitation models in an attempt to address the brain-injured person's lifelong needs. It is observed the actual practice of the espoused approach appears to vary between different rehabilitation settings. Each rehabilitation service is not only influenced by the client's needs, but also by the economic rationalisation of service provision funds. The organisation's need to be able to demonstrate effective outcomes with limited funds is central to the development of models and service delivery. In response to these listed influences, a model of providing targeted rehabilitation at different time periods is practised by the Epworth Hospital's community brain injury programs. This model attempts to provide input at the time the person prepares to address the transition points in their life. This paper aims to explore and to demonstrate the model developed by the Epworth community programs. This description will highlight how the targeted input of rehabilitation is practised, how the services are structured to provide the service, and how the costs are managed. A number of case studies will be presented to demonstrate the lifelong stages of input, the lifetime model, and the costeffectiveness of the program. 


\section{Family Participation in the Rehabilitation of the Child with Traumatic Brain Injury}

Lucia Braga

SARAH Network of Rehabilitation Hospitals, Brasilia, Brazil

The objective of this paper is to evaluate if children with traumatic brain injury (TBI) present better motor and cognitive outcomes during the period of one year when treated exclusively by professionals compared with children predominantly treated by the family under the supervision of a team. A study was conducted with randomised controlled trials using the evidence-based approach. One hundred and eighty children, 5-10 years of age at the study's onset, with injuries which had occurred 6 months $-2 \frac{1}{2}$ years earlier, were randomised and submitted to two different treatment approaches during one year. Group A were 74 children predominantly rehabilitated by the family. The parents underwent intensive training and performed the activities at home with the child, returning to the hospital bi-weekly for new orientations. Group B were 71 children who, during one year, attended daily rehabilitation sessions at the hospital and were exclusively treated by professionals. The children were assessed by the Weschler Intelligence Scale for Children and the Sarah Scale of Motor Development before and one year after starting treatment. Two-tailed Chi-square and Mann-Whitney tests and logistic-regression assessment revealed that the children in group A obtained better outcomes in the motor and the cognitive development scales than group B $(p<.05)$. The parents' educational levels showed no statistically significant bearing. Thus the children with TBI who received development and cognitive stimulation from the family under the supervision of a specialised team presented better progress than those treated exclusively by professionals. These results point to a new perspective in the rehabilitation of these children, based on family participation.

\section{Closing Address: Cognitive Rehabilitation and Emotion}

Huw Williams

School of Psychology, University of Exeter, Exeter, UK

Cognitive rehabilitation (CR) was developed to enable brain injured sur$\checkmark$ vivors to develop awareness and acceptance of their cognitive deficits so as to develop strategies to compensate for them and regain social roles (Wilson, 1997). There has been concern over whether CR can be effective in targeting real world behaviour change. One major reason for this is that the "laboratory" style environments in which training takes place may not fully "match" the real world, where people interact with others in fluid, emotionally-toned exchanges. The social world is laden with barely perceptible behavioural and emotional cues for guiding behaviour. Survivors of brain injury often have difficulties processing such stimuli, and may have problematic exchanges leading to disengagement from society and, often, severe mental health problems. In a recent development in this field, Williams, Evans and Wilson (2003a, 2003b) have shown that such survivors often suffer chronic mental health problems. They have described cases in which CR was used in combination with cognitive behaviour therapy (CBT) for enabling survivors to engage in rehabilitation, manage affect, and achieve personally meaningful goals. They described cases of survivors having both cognitive (attention and memory disorders and poor insight) 
and psychological disorders (posttraumatic stress disorder, depression and alcohol misuse, and/or obsessive-compulsive disorder) who were treated by such a combination of interventions, which led to significant improvements in their domestic, social and vocational functioning. In this paper, Williams will argue that there is a need, and opportunity, for improving models of neuropsychological functioning by attending to such affective elements of cognition, and will present data from ongoing studies in brain injury on factors such as mood, sleep, pain, coping styles and relationships status which provide a basis for a framework for integrating cognitive, affective and social factors in $\mathrm{CR}+\mathrm{CBT}$. 